\title{
Design of Multistage Air Cleaning Device for Bus
}

\author{
Mengjie $\mathrm{Wu}^{1 *}$, Shuang $\mathrm{Hu}^{1}$, Yuheng Tang ${ }^{1}$ \\ ${ }^{1}$ School of Energy and Power Engineering, Wuhan University of Technology, Wuhan, Hubei, 430063, China
}

\begin{abstract}
According to the survey, the concentration of pollutants in most buses exceeds the standard seriously, which not only reduces the comfort of passengers, but also harms the health of drivers and passengers. Therefore, it is very important to purify the air in the bus. However, there are many kinds of air pollutants in the bus, and the effect of single purification method is poor. Through the reasonable combination of activated carbon, photocatalyst and negative oxygen ions, the device can absorb and filter organic pollutants, bacteria and viruses in the air. Therefore, the device realizes the treatment of a variety of air pollutants in the bus, effectively improves the air quality in the bus, and also provides a healthy and comfortable travel environment for passengers.
\end{abstract}

\section{Introduction}

With the increase of people's awareness of energy saving and emission reduction, people will give priority to taking buses, but most existing buses have serious air problems during operation. The most common problems are air odors, germs, viruses and organic pollutants [1]. Air cannot be renewed and purified for a long time. Poor air quality in vehicles not only affects the physical and mental health of passengers, but also causes mental fatigue among drivers and flight attendants, and may even lead to serious safety accidents. As a result, many people choose to drive in private cars, making per capita carbon emissions high.

\section{Structure design}

According to the ventilation system of existing buses, the air conditioner in the car is installed at the center of the top of the car, and the external air is transported into the car through the air ducts at the two ends of the car. Only when the air is exchanged, the gas enters from the outside. When the air is cooled and heated, the gas circulates internally. Therefore, the device is installed with a fibrous activated carbon filter, a photocatalyst filter, and an anion generator in the position of the air intake main pipe of the air conditioner in this order. The fibrous activated carbon filter and photocatalyst filter purify the internal circulating air or external air. The negative oxygen ions generated by the negative ion generator are transported into the cabin along with the air duct to purify the indoor air. The following is the detailed design of each part.

\subsection{Purification photocatalyst \\ structure \\ design \\ of}

Photocatalyst is a kind of catalyst with photocatalytic function represented by nanometer titanium dioxide. It has strong oxidizing ability. It can decompose a large amount of organic or inorganic pollutants into carbon dioxide and water by the catalysis of light source. The functions of mold, anti-fouling and self-cleaning, and air purification will not change or lose itself when the catalytic reaction is performed. It will continuously purify pollutants under the irradiation of light for a long duration of action, high efficiency, safety, non-toxicity and no pollution.

Short-wave UV light has a strong direct photolysis effect on organic matter. The closer the dominant wavelength of an ultraviolet lamp is to the characteristic wavelength of the maximum absorption peak of an organic substance, the better the photolysis of the organic substance. In view of the fact that most of the maximum absorption peak or the second largest absorption peak of organic matter is located at $200-300 \mathrm{~nm}$ [2], and currently the ultraviolet wavelengths on the market are divided into two types of $253.7 \mathrm{~nm}$ and $365 \mathrm{~nm}$, so we choose an ultraviolet lamp with a main wavelength of $253.7 \mathrm{~nm}$.

The catalytic rate of titanium dioxide is different under different light intensities. As shown in Figure 1, it can be seen that the maximum values of the photocatalytic decomposition rate of formaldehyde at $15 \mathrm{~W}, 30 \mathrm{~W}$, and $60 \mathrm{~W}$ are $32.80 \%, 68.81 \%$, and $77.85 \%$ [3].

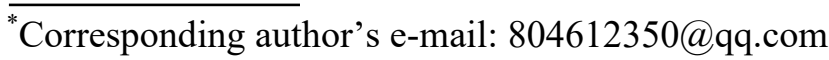




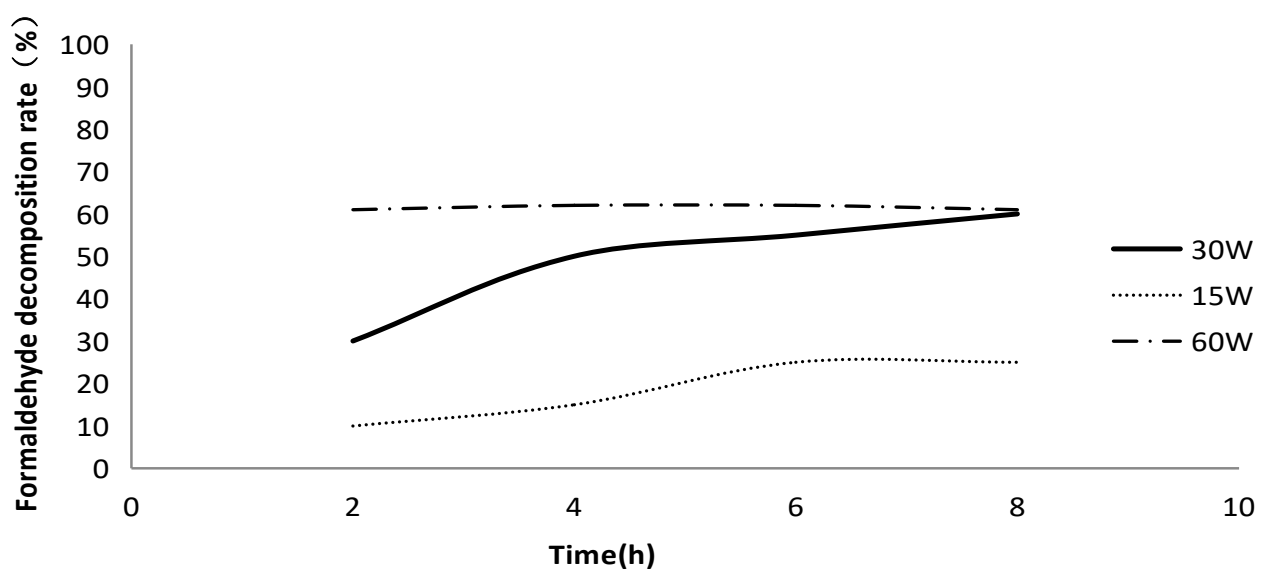

Figure 1. Photocatalytic degradation rate of VOCs in cars at different times

Based on the above two points, this device is planned to use U-shaped UV lamps of $12 \mathrm{~V}, 30 \mathrm{~W}$ and $253.7 \mathrm{~nm}$.

Time is also an important factor affecting the degradation efficiency of photocatalysts. As shown in Figure 2. In a short period of time, the gas molecules and the catalyst are too late to contact, which is not conducive to the adsorption of the reactants, and also accelerates the desorption of the intermediate products. Deep oxidation can further increase the degradation rate.

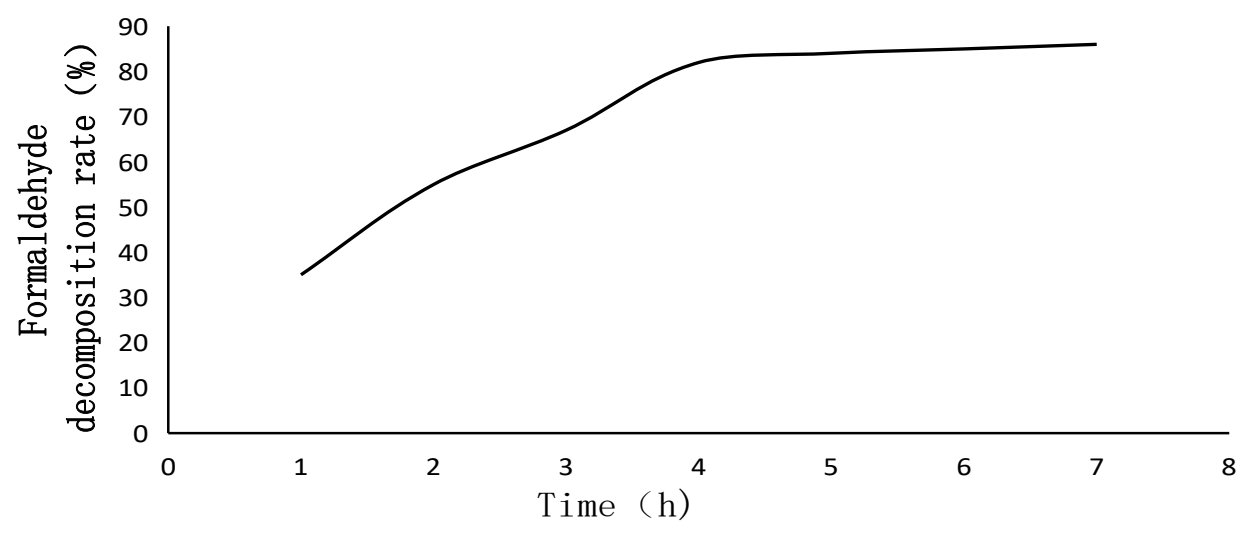

Figure 2. Photocatalytic degradation rate of VOCs in cars at different times

Accordingly, multi-level structure, the first stage is a fiber activated carbon filter, activated carbon adsorption capacity, can effectively filter adsorb contaminants, so that the concentration of molecules surrounding the titanium dioxide layer adhering to the sieve is increased, thereby enhancing the photocatalytic efficiency the second stage is a nickel-based photocatalyst filter the photocatalyst fixed on a substrate surface, and to ensure the firmness of the photocatalyst activity, Decomposition of organic pollutants ultraviolet lamp irradiated the substrate surface a specific wavelength band, and to destroy viruses and bacteria. When the entire layer structure is intended to contain air in the intake manifold of the air conditioner, gas exchange in the air conditioner, the indoor air conditioning input for effective cleaning [4].

\subsection{Design negative oxygen ions occurs}

Converting the discharge current via the input means of the negative ions to obtain a negative high voltage direct current, and then by a corona discharge electrode at high speed a large number of electrons emitted, electrons are immediately incorporated oxygen in the air and water vapor molecules form negative oxygen ions, negative oxygen ions can improve a person's sleep, promote metabolism, enhance human disease; groups may be neutralized to settle in the air respirable particulate matter, dust, and other organic pollutants; negative oxygen ions having further strong reduction, the air can kill harmful bacteria and viruses, so you can achieve the effect of improving air quality.

At present, the power range of negative ion generators on the market is generally less than $10 \mathrm{~W}$. The power and working current required by this device are very low. It is easy to design the structure of the device, so that it can be used in public transportation such as buses to optimize the air of buses. Good effect of quality. 


\section{Air purification function}

\subsection{Photocatalyst media filter filters the air entering the car.}

The Photocatalyst filter arranged in the air intake duct of the air conditioner can purify the outside air that is drawn into the car when it is illuminated by LED lights, while removing the outside air harmful gases in the air, such as formaldehyde, nitric oxide, carbon monoxide, etc ; effectively kill a variety of harmful bacteria in the outside air, can break down the toxins released by bacteria or fungi and do harmless treatment; have deodorization, anti-fouling. It can remove the odor of the outside air and purify the air entering the car [5].

\subsection{Negative oxygen ion generator to purify the air in the car.}

The negative ion generator arranged at the air outlet of the air conditioner can deliver negative oxygen ions to the car while supplying air. The strong reducing property of negative oxygen ions can kill harmful bacteria in the air inside the car, reducing the number of germs transmitted by air; inhalation of air containing negative oxygen ions can improve the health of the human body; negative oxygen ions can also combine with positively charged dust and dust to settle and react with harmful gases to produce $\mathrm{CO}_{2}$ and water.

\section{Simulation analysis}

\subsection{Structural design and concentration simulation analysis of negative oxygen ion generation.}

After investigation, the most widely used bus model in Wuhan is the G2 series WG6100. The specific parameters of the bus are: $10500 \times 2500 \times 3200(\mathrm{~mm})$. The theoretical maximum ventilation speed of the ventilator is $25 \mathrm{~m}^{3} / \mathrm{min}$. The air outlets of the air conditioning system are distributed on the roof of the passenger seat through the air duct, and the air volume is adjusted by the driver [6]. This project intends to simulate the distribution of fluid pressure and speed in the vehicle under different working conditions, as well as the content of negative oxygen ions at different locations in the vehicle.

Firstly, simulate the air flow when the window is closed and the air conditioner is closed under different air supply speeds. At this time, the air-conditioning air supply speed in the cabin is relatively low, and the space in the cabin is small. The effects of forced convection and natural convection need to be considered. Because the temperature is unevenly distributed in height, local flow occurs under the action of buoyancy. At the same time, there are many seats and passengers in the car. The air flow in the car is very complex. Calculate the Reynolds pressure and flow rate, and use the Realizable $\mathrm{k}-\varepsilon$ model to simulate the complex flow of air in the car. At the same time, for the sake of simplicity, the following assumptions are made: the flow in the compartment is considered as a steady state process; the air in the compartment is considered incompressible; except for the ventilator, the compartment is well sealed. The SIMPLE algorithm is applied to the model to solve the discrete governing equations. In this way, the air flow in the compartment at different air speeds is obtained.

Secondly, the distribution of the concentration of negative ions generated by the negative ion generator in the entire compartment is simulated. The common flow of negative ions and air can be regarded as two-phase flow, and the flow of fluid in the pipeline can be regarded as laminar flow, but its flow in the compartment is very complicated. It is regarded as turbulence as in the first case and the Realizable $\mathrm{k}-\varepsilon$ model is used. Simulate the negative ion concentration distribution of the entire cabin under different powers of the negative ion generator, so as to obtain the optimal working power of the negative ion generator, so that the negative ion concentration in most areas of the cabin is maintained at about 1 million per $\mathrm{cm}^{3}$.

\subsection{Simulation results of experimental models}

First, the experimental model and problems are simplified. The flow in the model is regarded as a steady state process, and the air is regarded as an incompressible flow. The compartment is well sealed except for the air inlet and outlet. And simplify some complicated curved surfaces that do not affect the air flow in the car, and get a simulation model.

The model is then meshed. As shown in Figure 3, the total number of meshes in the model is 585704 , and the minimum boundary size is $4 * 10^{\wedge}(-5)$ meters. The partitioning method uses automatic partitioning to adapt to changing meshes. Size and area shape characteristics. The checked grid quality is good, and it is also convenient for subsequent calculations. 


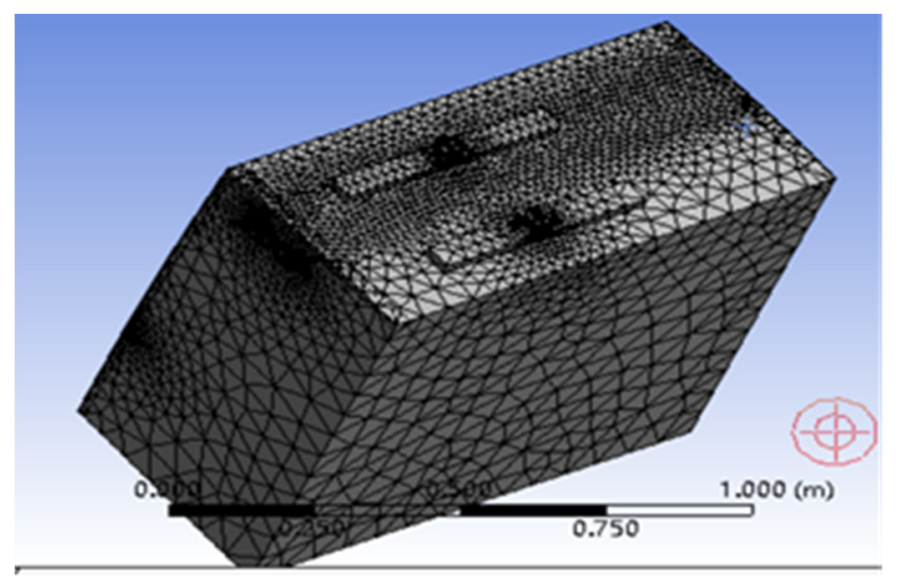

Figure 3. Meshing diagram

The air supply speed of the air conditioner in the cabin is relatively low, and the space in the cabin is small. The effects of forced convection and natural convection need to be considered. The distribution is uneven in height, and local flow occurs under the action of buoyancy. The air flow in the vehicle is very complicated. It is a typical turbulent flow. You can use the turbulence model in FLUENT to calculate the flow velocity and use the standard $\mathrm{k}-\varepsilon$ model. The internal air complex flow is calculated, and after consulting relevant papers, it is found that the model fits well in this case. The inlet is set as a speed inlet. The diameter of the blower used in the experiment is $100 \mathrm{~mm}$, and the maximum ventilation volume per hour is 130 cubic meters. The wind speed near it is calculated to be $3 \mathrm{~m} / \mathrm{s}$, which is the wind speed at the entrance, and the direction is perpendicular to the boundary inward [7]. The outlet is set as a pressure outlet, and the pressure at the outlet is set to zero as a relative reference point. Later, the SIMPLE algorithm was used to solve the discrete governing equations, and the relaxation factor was adjusted to help convergence. After 500 iterations, the residual plot was observed to find that the results had converged.

Finally, analyze the results and calculate the velocity vector cloud diagram of the interior and the boundary, as shown in Figure 4. Observing the image shows that the velocity near the entrance and exit is relatively large at about $3 \mathrm{~m} / \mathrm{s}$, the maximum speed is $5.7 \mathrm{~m} / \mathrm{s}$, and the minimum speed is $0.02 \mathrm{~m} / \mathrm{s}$. Uninterrupted air circulation is performed throughout the model, and the air velocity on the other side of the model air outlet is low. Then observe the X-Y plot, as shown in Figure 5, and find that the speed gradually decreases from the top to the bottom, and the flow at the bottom of the model is weak [8].

The overall flow of the model is good and meets expectations. Effective air circulation and air purification can be performed in the model.

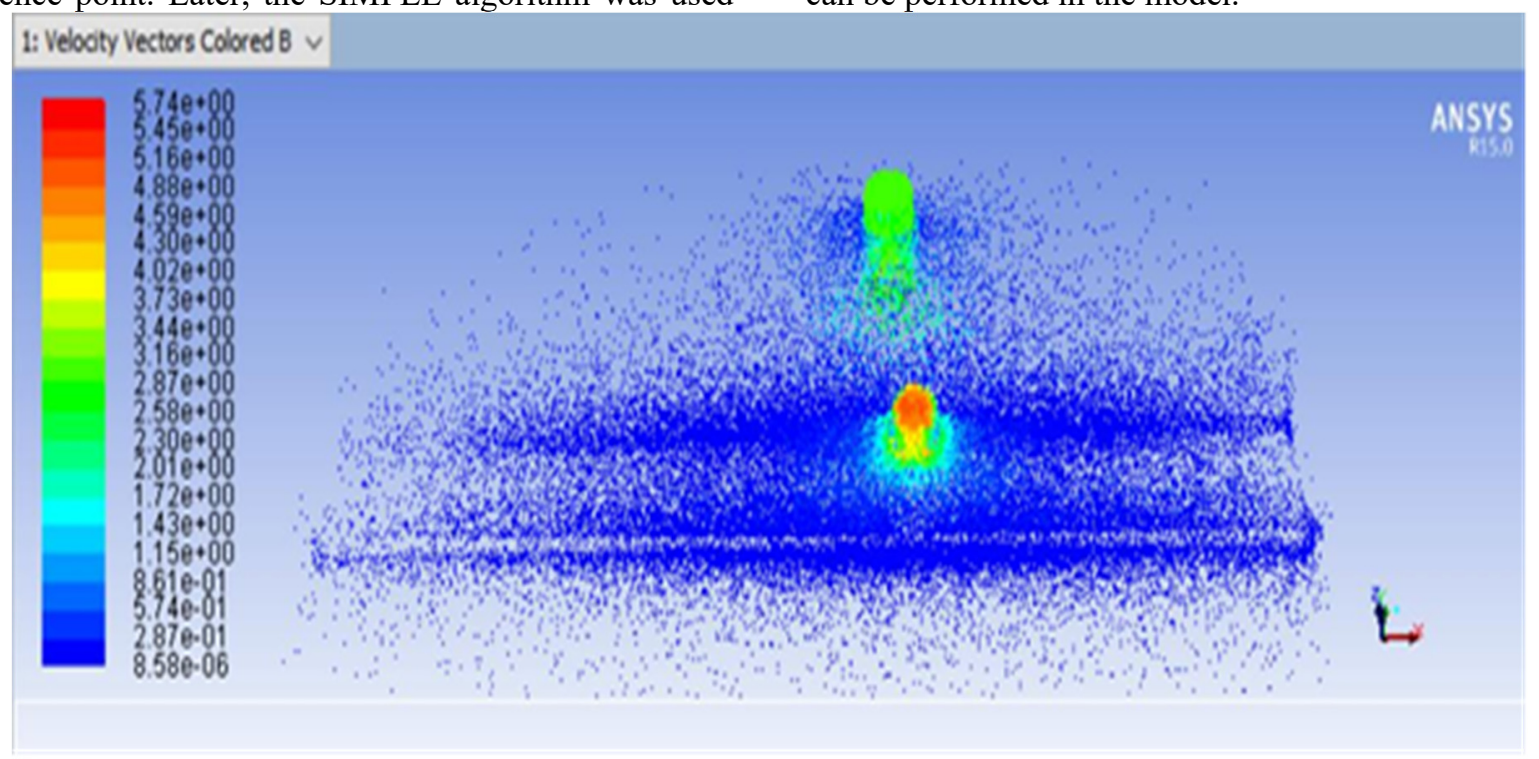

Figure 4. Velocity Vector Cloud 


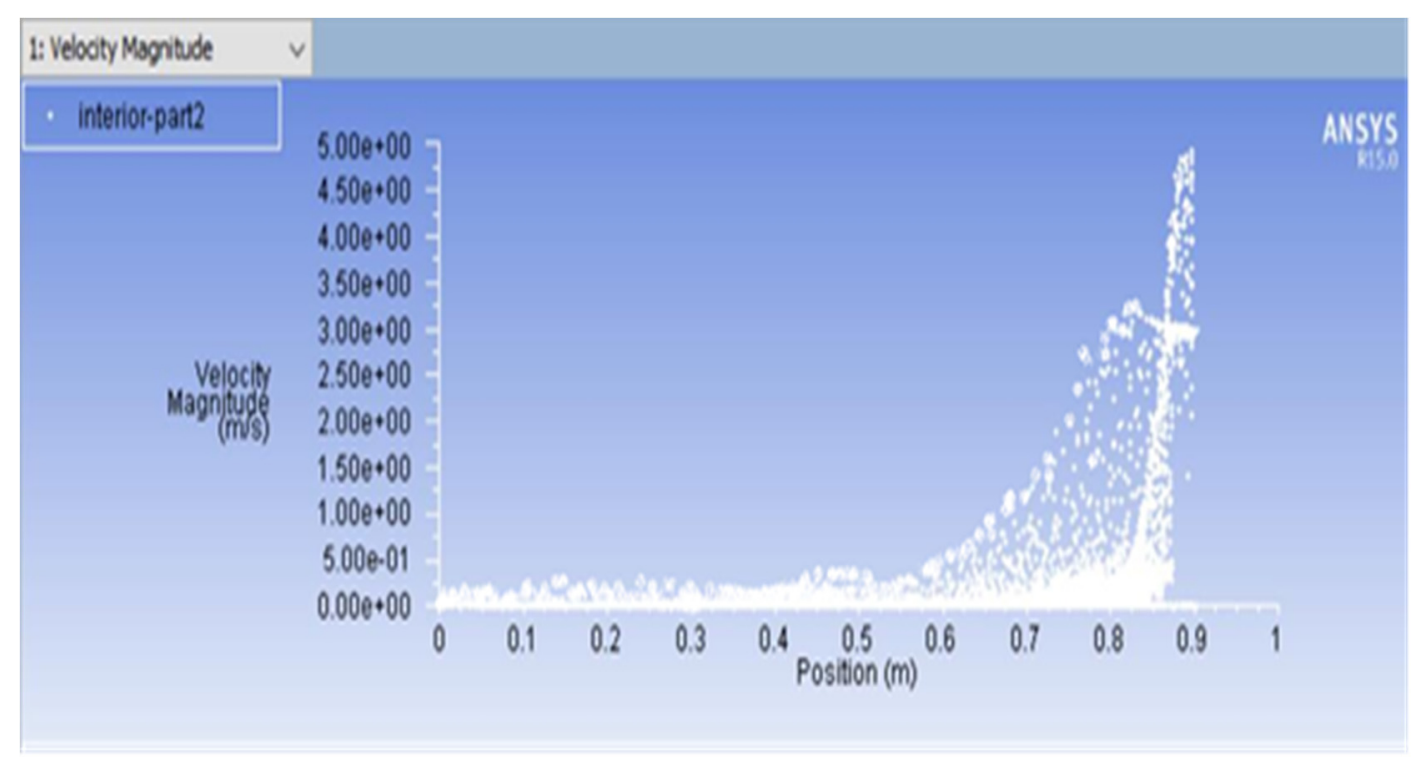

Figure 5. Speed-altitude data

\section{Conclusion}

According to the actual conditions in the bus and the specific pollutant types, the device combines the characteristics of fiber activated carbon filter, photocatalyst technology and negative ion generation technology to perform multi-stage purification of the air in the bus and effectively control the concentration of pollutants in the bus. In combination with the existing bus ventilation system, the negative ion generating device is installed at the air inlet duct of the air conditioner, so that the negative oxygen ions enter the car with the air conditioner, which achieves the purpose of completing air purification while taking in air.

In the future, it is expected that the multi-stage air purification system applicable to buses will be upgraded and retrofitted so that it can be applied to different modes of transportation. Large public transportation such as long-distance buses and luxury buses can be combined with its central air-conditioning system. Nowadays, the manufacturing of buses has gradually shifted its focus from power development to improving passenger comfort. This project fits well with the future development trend of buses. It can replace the current traditional sterilization and disinfection methods of buses, saving a lot of human and material resources. By improving the comfort of public transport, more people will give up taking private cars as the mode of travel, and choose to travel by public transport. This will not only reduce the current traffic congestion pressure, but also reduce a lot of noise pollution caused by private cars and a large consumption of energy. The device effectively solves the problem of environmental pollution caused by a large number of exhaust gas from private cars, and plays an important role in alleviating the global warming caused by excessive greenhouse gases.

\section{Acknowledgments}

Project Funding: National innovation and entrepreneurship training program for college students: 201810497147.

\section{References}

1. Tang, W. H., Ke, R. W., Gong, Z. H. (2011). Test investigation and improvement suggestions for air quality in Wuhan air-conditioned buses. J. Refrigeration, air-conditioning and electric machinery, 03-15.

2. Zhu, Y. (2011). Study on Photocatalytic Purification of Harmful Microorganisms in Central Air Conditioning System. D. Shenyang Jianzhu University .

3. Xie, C. P., Wang, S. G., Zhou, C. H., et al. (2011), Analysis of Factors Affecting the Degradation of Formaldehyde by $\mathrm{TiO} 2$ Photocatalyst. J. Journal of Tianjin Institute of Urban Construction. (02).

4. Zheng, S. Q., Huo, Z. (2010). Low-carbon urban spatial structure: A study from the perspective of private car travel .World Economic Papers .12-05.

5. Light Vehicle Pollutant Emission Limits and Measurement Methods. (2013). (China's Fifth Stage) GB 18352.5-2013.

6. Xu, Y., Zheng C., Liu, Z., et al. (2013). Electrostatic precipitation of airborne bioaerosols. Journal of Electrostatics, 71 (3).

7. Hanley, J. T., Ensor, D. S, Smith, D. D., et al. (2010). Fractional Aerosol Filtration Efficiency of In - Duct Ventilation Air Cleaners. Indoor Air, $4(3)$.

8. Li, L., Xu, Z., Song, C. et al. (2006). Adsorption-filtration characteristics of meltblown polypropylene fiber in purification of reclaimed water. Desalination,201 (1) : 198206. 